\title{
The Equational Theory of Weak Complete Simulation Semantics over BCCSP ${ }^{\star}$
}

\author{
Luca Aceto ${ }^{1,3}$, David de Frutos-Escrig 2,3 , Carlos Gregorio-Rodríguez ${ }^{2,3}$, and \\ Anna Ingólfsdóttir ${ }^{1,3}$ \\ 1 ICE-TCS, School of Computer Science, Reykjavik University, Iceland \\ 2 Departamento de Sistemas Informáticos y Computación, \\ Universidad Complutense de Madrid, Spain \\ 3 Abel Extraordinary Chair (Universidad Complutense-Reykjavik University)
}

\begin{abstract}
This paper presents a complete account of positive and negative results on the finite axiomatizability of weak complete simulation semantics over the language BCCSP. We offer finite (un)conditional groundcomplete axiomatizations for the weak complete simulation precongruence. In sharp contrast to this positive result, we prove that, in the presence of at least one observable action, the (in)equational theory of the weak complete simulation precongruence over BCCSP does not have a finite (in)equational basis. In fact, the collection of (in)equations in at most one variable that hold in weak complete simulation semantics over BCCSP does not have an (in)equational basis of 'bounded depth', let alone a finite one.
\end{abstract}

\section{Introduction}

Process algebras, such as ACP [4, 6], CCS [16] and CSP [13], are prototype specification languages for reactive systems. Such languages offer a small, but expressive, collection of operators that can be combined to form terms that describe the behaviour of reactive systems.

Since the seminal work by Bergstra and Klop [6], and Hennessy and Milner [12], the search for (in)equational axiomatizations of notions of behavioural semantics for fragments of process algebras has received much attention in concurrency theory. A complete axiomatization of a behavioural semantics yields a purely syntactic and model-independent characterization of the semantics of

\footnotetext{
* Luca Aceto and Anna Ingólfsdóttir have been partially supported by the projects 'New Developments in Operational Semantics' (nr. 080039021) and 'Meta-theory of Algebraic Process Theories' (nr. 100014021) of the Icelandic Research Fund. David de Frutos-Escrig and Carlos Gregorio-Rodríguez have been partially supported by the Spanish projects TESIS (TIN2009-14312-C02-01), DESAFIOS10 (TIN2009-14599C03-01) and PROMETIDOS S2009/TIC-1465. The paper was begun when David de Frutos-Escrig and Carlos Gregorio-Rodríguez held Abel Extraordinary Chair positions at Reykjavik University, and finalized while Luca Aceto and Anna Ingolfsdottir held Abel Extraordinary Chairs at Universidad Complutense de Madrid, Spain, supported by the NILS Mobility Project.
} 
a process algebra, and paves the way to the application of theorem-proving techniques in establishing whether two process descriptions exhibit related behaviours.

The aim of this paper is to contribute to the study of the equational theory of process algebras modulo notions of semantics that abstract, in some suitable fashion, from internal computations in the behaviour of processes. In [2], we provided a complete account of positive and negative results on the finite axiomatizability of weak simulation $[15,19]$ and weak ready simulation semantics $[7,14]$ over the language BCCSP. (This language contains only the basic process algebraic operators from CCS [16] and CSP [13], but is sufficiently powerful to express all finite synchronization trees [16].) In the present paper, we focus on the study of the equational theory of weak complete simulation semantics. Weak complete simulation is a deadlock-sensitive variation on simulation, which is the 'weak counterpart' of complete simulation [11]. Our definition of the notion of weak complete simulation is based on considering a process 'complete', or 'mute', when it cannot perform any observable action. For instance, letting the symbol $\tau$ denote an unobservable action [16], the process $\tau$ is mute, but neither $\tau a$ nor $\tau+a$ is.

We offer finite conditional and unconditional ground-complete axiomatizations for the weak complete simulation precongruence. (An (in)equational axiomatization is called ground-complete if it can prove all the valid (in)equivalences relating terms with no occurrences of variables in the process algebra of interest.) In sharp contrast to this positive result, we prove that, in the presence of at least one observable action, the (in)equational theory of the weak complete simulation precongruence over BCCSP does not have a finite (in)equational basis. In fact, the collection of (in)equations in at most one variable that hold true in weak complete simulation semantics over BCCSP does not have an (in)equational basis of 'bounded depth', let alone a finite one.

Our work contributes to the extension to the 'weak' setting, where processes may perform transitions labelled with the unobservable action $\tau$, of a collection of non-trivial results that have been obtained for behavioural semantics that consider each action processes perform as being observable by their environment. Our positive results build on, for instance, the encyclopedic studies presented in $[9,11]$. In particular, we use conditional axioms to provide a simple and clear picture of the (in)equalities that are valid in weak complete simulation semantics, and derive purely equational ground-complete axiomatizations from conditional axiomatizations in a rather uniform fashion. On the other hand, our negative results are shown using proof-theoretic techniques that have their root in, e.g., the seminal journal paper [8]. (The article [3] surveys classic proof techniques for showing non-finite axiomatizability results.)

The paper is organized as follows. Section 2 presents the syntax and the operational semantics for the language BCCSP, and reviews the necessary background on (in)equational logic as well as classic axiom systems for strong bisimulation equivalence and observational congruence (the largest congruence included in weak bisimulation equivalence). Section 3 is devoted to our positive 
and negative results on the finite axiomatizability of the weak complete simulation preorder. We conclude the paper by discussing further related work and directions for future research in Section 4.

Due to lack of space, we have preferred to omit the proofs of our results. Proofs as well as further details and explanations can be found in Section 4 of the extended paper that is available at the URL http://www.hr.is/faculty/ luca/PAPERS/OnWeakSemantics . pdf.

\section{Preliminaries}

To set the stage for the developments offered in the rest of the paper, we present the syntax and the operational semantics for the language BCCSP, some background on (in)equational logic, and classic axiom systems for strong bisimulation equivalence and observational congruence [16].

Syntax of BCCSP $\operatorname{BCCSP}\left(A_{\tau}\right)$ is a basic process algebra for expressing finite process behaviour. Its syntax consists of closed (process) terms $p, q, r$ that are constructed from a constant $\mathbf{0}$, a binary operator ${ }_{-}+_{-}$called alternative composition, or choice, and unary prefix operators $\alpha_{-}$, where $\alpha$ ranges over some set $A_{\tau}$ of actions of the form $A \cup\{\tau\}$, where $\tau$ is a distinguished action symbol that is not contained in $A$. Following Milner [16], we use $\tau$ to denote an internal, unobservable action of a reactive system, and we let $a, b, c$ denote typical elements of $A$ and $\alpha$ range over $A_{\tau}$. The set of closed terms is named $\mathrm{T}\left(\operatorname{BCCSP}\left(A_{\tau}\right)\right)$, in short $\mathrm{T}\left(A_{\tau}\right)$. We write $|A|$ for the cardinality of the set of observable actions.

Open terms $t, u, v$ can moreover contain occurrences of variables from a countably infinite set $V$ (with typical elements $x, y, z)$. We use $\mathbb{T}\left(\operatorname{BCCSP}\left(A_{\tau}\right)\right.$ ), in short $\mathbb{T}\left(A_{\tau}\right)$, to denote the set of open terms. The depth of a term $t$ is the maximum nesting of prefix operators in $t$.

In what follows, for each non-negative integer $n$ and term $t$, we use $a^{n} t$ to stand for $t$ when $n=0$, and for $a\left(a^{n-1} t\right)$ otherwise. As usual, trailing occurrences of $\mathbf{0}$ are omitted; for example, we shall usually write $\alpha$ in lieu of $\alpha \mathbf{0}$.

A (closed) substitution maps variables in $V$ to (closed) terms. For every term $t$ and substitution $\sigma$, the term $\sigma(t)$ is obtained by replacing every occurrence of a variable $x$ in $t$ by $\sigma(x)$. Note that $\sigma(t)$ is closed if $\sigma$ is a closed substitution.

Transitions and their defining rules Intuitively, closed $\operatorname{BCCSP}\left(A_{\tau}\right)$ terms represent finite process behaviours, where $\mathbf{0}$ does not exhibit any behaviour, $p+q$ is the nondeterministic choice between the behaviours of $p$ and $q$, and $\alpha p$ executes action $\alpha$ to transform into $p$. This intuition is captured, in the style of Plotkin [20], by the simple transition rules below, which give rise to $A_{\tau}$-labelled transitions between closed terms.

$$
\underset{\alpha x \stackrel{\alpha}{\longrightarrow} x}{\frac{x \stackrel{\alpha}{\longrightarrow} x^{\prime}}{x+y \stackrel{\alpha}{\longrightarrow} x^{\prime}}} \quad \frac{y \stackrel{\alpha}{\longrightarrow} y^{\prime}}{x+y \stackrel{\alpha}{\longrightarrow} y^{\prime}}
$$


The operational semantics is extended to open terms by assuming that variables do not exhibit any behaviour.

The so-called weak transition relations $\stackrel{\alpha}{\Longrightarrow}\left(\alpha \in A_{\tau}\right)$ are defined over $\mathbb{T}\left(A_{\tau}\right)$ in the standard fashion as follows.

- We use $\stackrel{\tau}{\Longrightarrow}$ for the reflexive and transitive closure of $\stackrel{\tau}{\longrightarrow}$.

- For each $a \in A$ and for all terms $t, u \in \mathbb{T}\left(A_{\tau}\right)$, we have that $t \stackrel{a}{\Longrightarrow} u$ if, and only if, there are $t_{1}, t_{2} \in \mathbb{T}\left(A_{\tau}\right)$ such that $t \stackrel{\tau}{\Longrightarrow} t_{1} \stackrel{a}{\longrightarrow} t_{2} \stackrel{\tau}{\Longrightarrow} u$.

Preorders and their kernels We recall that a preorder $\precsim$ is a reflexive and transitive relation. Let $\precsim$ be a preorder over the set of closed terms $\mathrm{T}\left(A_{\tau}\right)$. For terms $t, u \in$ $\mathbb{T}\left(A_{\tau}\right)$, we define $t \precsim u$ if, and only if, $\sigma(t) \precsim \sigma(u)$ for each closed substitution $\sigma$.

The kernel $\approx$ of a preorder $\precsim$ is the equivalence relation it induces, and is defined thus:

$$
t \approx u \text { if, and only if, }(t \precsim u \text { and } u \precsim t) .
$$

It is easy to see that the kernel of a preorder $\precsim$ is the largest symmetric relation included in $\precsim$.

Inequational logic An inequation (respectively, an equation) over the language $\operatorname{BCCSP}\left(A_{\tau}\right)$ is a formula of the form $t \leq u$ (respectively, $t=u$ ), where $t$ and $u$ are terms in $\mathbb{T}\left(A_{\tau}\right)$. An (in)equational axiom system is a set of (in)equations over the language $\operatorname{BCCSP}\left(A_{\tau}\right)$. An equation $t=u$ is derivable from an equational axiom system $E$, written $E \vdash t=u$, if it can be proven from the axioms in $E$ using the rules of equational logic (viz. reflexivity, symmetry, transitivity, substitution and closure under $\operatorname{BCCSP}\left(A_{\tau}\right)$ contexts).

$$
\begin{gathered}
t=t \quad \frac{t=u}{u=t} \quad \frac{t=u \quad u=v}{t=v} \\
\frac{t=u}{\sigma(t)=\sigma(u)} \quad \frac{t=u}{\alpha t=\alpha u} \quad \frac{t=u t^{\prime}=u^{\prime}}{t+t^{\prime}=u+u^{\prime}}
\end{gathered}
$$

For the derivation of an inequation $t \leq u$ from an inequational axiom system $E$, the rule for symmetry - that is, the second rule above-is omitted. We write $E \vdash t \leq u$ if the inequation $t \leq u$ can be derived from $E$.

It is well known that, without loss of generality, one may assume that substitutions happen first in (in)equational proofs, i.e., that the fourth rule may only be used when its premise is one of the (in)equations in E. Moreover, by postulating that for each equation in $E$ also its symmetric counterpart is present in $E$, one may assume that applications of symmetry happen first in equational proofs, i.e., that the second rule is never used in equational proofs. (See, e.g., [8, page 497] for a thorough discussion of this notion of 'normalized equational proof'.) In the remainder of this paper, we shall always tacitly assume that equational axiom systems are closed with respect to symmetry. Note that, with this assumption, there is no difference between the rules of inference of equational 
and inequational logic. In what follows, we shall consider an equation $t=u$ as a shorthand for the pair of inequations $t \leq u$ and $u \leq t$.

The depth of $t \leq u$ and $t=u$ is the maximum of the depths of $t$ and $u$. The depth of a collection of (in)equations is the supremum of the depths of its elements. So, the depth of a finite axiom system $E$ is zero, if $E$ is empty, and it is the largest depth of its (in)equations otherwise.

An inequation $t \leq u$ is sound with respect to a given preorder relation $\precsim$ if $t \precsim u$ holds. An (in)equational axiom system $E$ is sound with respect to $\precsim$ if so is each (in)equation in $E$.

Classic axiomatizations for notions of bisimilarity The well-known axioms $B_{1}-B_{4}$ for $\operatorname{BCCSP}\left(A_{\tau}\right)$ given below stem from [12]. They are $\omega$-complete [18], and sound and ground-complete $[12,16]$, over $\operatorname{BCCSP}\left(A_{\tau}\right)$ (over any non-empty set of actions) modulo bisimulation equivalence $[16,19]$, which is the finest semantics in van Glabbeek's spectrum [11].

$$
\begin{array}{rlrl}
B_{1} & x+y & =y+x \\
B_{2} & (x+y)+z & =x+(y+z) \\
B_{3} & x+x & =x \\
B_{4} & x+\mathbf{0} & =x
\end{array}
$$

In what follows, for notational convenience, we consider terms up to the least congruence generated by axioms $B_{1}-B_{4}$, that is, up to bisimulation equivalence. We use summation $\sum_{i=1}^{n} t_{i}$ (with $n \geq 0$ ) to denote $t_{1}+\cdots+t_{n}$, where the empty sum denotes $\mathbf{0}$. Modulo the equations $B_{1}-B_{4}$ each term $t \in \mathbb{T}\left(A_{\tau}\right)$ can be written in the form $\sum_{i=1}^{n} t_{i}$, where each $t_{i}$ is either a variable or is of the form $\alpha t^{\prime}$, for some action $\alpha$ and term $t^{\prime}$.

In a setting with internal transitions, the classic work of Hennessy and Milner on weak bisimulation equivalence and on the largest precongruence included in it, observational congruence, shows that the axioms $B_{1}-B_{4}$ together with the axioms $W_{1}-W_{3}$ below are sound and complete over BCCSP $\left(A_{\tau}\right)$ modulo observational equivalence. (See $[12,16,17]$.

$$
\begin{aligned}
W_{1} & \alpha x & =\alpha \tau x \\
W_{2} & \tau x & =\tau x+x \\
W_{3} & \alpha(\tau x+y) & =\alpha(\tau x+y)+\alpha y
\end{aligned}
$$

The above axioms are often referred to as the $\tau$-laws. For ease of reference, we write

$$
B W=\left\{B_{1}, B_{2}, B_{3}, B_{4}, W_{1}, W_{2}, W_{3}\right\} .
$$

\section{Weak Complete Simulation}

In the remainder of this paper, we study the notion of complete simulation preorder in a setting with $\tau$ actions. Recall that, in the setting without $\tau$, a complete simulation [11] is a simulation relation that is only allowed to relate a state with no outgoing transitions to states with the same property. 
Definition 1. We say that process $p \in \mathrm{T}\left(A_{\tau}\right)$ must terminate (or is mute), written $p \Downarrow$, iff there does not exist any $a \in A$ such that $p \stackrel{a}{\Longrightarrow}$.

Note that $p$ is not mute, written $p \nVdash$, if, and only if, there exist $p^{\prime} \in \mathrm{T}\left(A_{\tau}\right)$, $n \geq 0$ and $a \in A$ such that $p(\stackrel{\tau}{\longrightarrow})^{n} p^{\prime} \stackrel{a}{\longrightarrow}$, where $(\stackrel{\tau}{\longrightarrow})^{n}$ denotes the $n$-fold composition of the relation $\stackrel{\tau}{\longrightarrow}$.

Definition 2. The weak complete simulation preorder, denoted by $\precsim_{C S}$, is the largest relation over terms in $\mathrm{T}\left(A_{\tau}\right)$ satisfying the following conditions whenever $p \precsim_{C S}$ q and $\alpha \in A_{\tau}$ :

- if $p \stackrel{\alpha}{\longrightarrow} p^{\prime}$ then there exists some term $q^{\prime}$ such that $q \stackrel{\alpha}{\Longrightarrow} q^{\prime}$ and $p^{\prime} \precsim C s q^{\prime}$, and

- if $p \Downarrow$ then $q \Downarrow$.

We say that $p, q \in \mathrm{T}\left(A_{\tau}\right)$ are weak complete simulation equivalent, written $p \approx_{C S}$ $q$, iff $p$ and $q$ are related by the kernel of $\precsim_{C S}$, that is when both $p \precsim_{C S} q$ and $q \precsim C S p$ hold.

It is easy to see that if $p \precsim C S q$ and $q \Downarrow$, then $p \Downarrow$.

Note that $\precsim_{C S}$ is not a precongruence with respect to the choice operator of $\operatorname{BCCSP}\left(A_{\tau}\right)$. Indeed, it is immediate to show that $\tau \mathbf{0} \precsim_{C S} \mathbf{0}$, but $\tau \mathbf{0}+a \mathbb{L}_{C S}$ $\mathbf{0}+a$.

Definition 3. We denote by $\sqsubseteq_{C S}$ the largest precongruence over $\mathrm{T}\left(A_{\tau}\right)$ included in $\precsim_{C S}$. Formally, $p \sqsubseteq \mathrm{CS} q$ iff

$-p \precsim C S q$,

- $p \precsim_{C S} q \Rightarrow \forall \alpha \in A_{\tau} \quad \alpha p \precsim_{C S} \alpha q$, and

$-p \precsim_{C S} q \Rightarrow \forall r \in \mathrm{T}\left(A_{\tau}\right) \quad p+r \precsim_{C S} q+r$.

The definition of the largest precongruence included in $\precsim C S$ is purely algebraic and difficult to use to study that relation. We next present a behavioural characterization of $\sqsubseteq C s$.

Definition 4. The preorder relation $\lesssim C S$ between processes in $\mathrm{T}\left(A_{\tau}\right)$ is defined as follows: $p \lesssim C S q$ iff

- $p \precsim C S q$, and

- whenever $p \stackrel{\tau}{\longrightarrow} p^{\prime}$ for some $p^{\prime}$ such that $p^{\prime} \Downarrow$, there exists some $q^{\prime}$ such that $q(\stackrel{\tau}{\longrightarrow})^{+} q^{\prime}$ and $q^{\prime} \Downarrow$.

We denote the kernel of $\lesssim_{C S}$ by $\bar{\sim}_{C S}$.

Example 1. It is immediate to see that $\tau \mathbf{0} \mathbb{L}_{C S} \mathbf{0}$. On the other hand, $\tau a \lesssim_{C S} a$ does hold because the second requirement in Definition 4 is vacuous. In general, $\tau p \lesssim C S p+q$ holds for all $p$ and $q$ provided that $p$ is not mute.

Proposition 1 (Behavioural characterization of $\sqsubseteq_{C S}$ ). $p \lesssim_{C S} q$ if, and only if, $p \sqsubseteq C S q$, for all $p, q \in \mathrm{T}\left(A_{\tau}\right)$. 


\subsection{Ground-complete axiomatizations}

In order to find a set of equations that gives a ground-complete axiomatization for the largest precongruence included in the weak complete simulation preorder, it is natural to consider the following (conditional) equations.

$$
\begin{array}{ll}
\left(C S_{\tau}\right) & (x \Downarrow \Leftrightarrow y \Downarrow) \Rightarrow x \leq x+y \\
\left(C S_{\tau e}\right) & \tau(a x+y)=a x+y
\end{array}
$$

The first equation, $C S_{\tau}$, is similar to the key axiom in the axiomatization for the complete simulation preorder in the concrete case, see e.g. [10]. However, in our setting, the mute predicate takes into account the silent steps of processes. This conditional equation restricts the applicability of inequation

$$
\text { (S) } x \leq x+y \text {, }
$$

which is only sound in (weak) complete simulation semantics when the terms substituted for the variables $x$ and $y$ have the same 'termination status'.

The second equation, $C S_{\tau e}$, is a restricted version of equation

$$
\text { ( } \tau e) \quad \tau x=x,
$$

which is valid in weak simulation semantics, but is unsound in weak complete simulation semantics. Intuitively, equation $C S_{\tau e}$ expresses the fact that a process of the form $\tau p$, for some term $p$ that is not mute, is weak complete simulation equivalent to $p$. In fact, equation $C S_{\tau e}$ could 'equivalently' be formulated as a conditional equation thus:

$$
x \Downarrow \Rightarrow \tau x=x .
$$

Proposition 2. The set of equations

$$
E_{C S \leq}^{c}=B W \cup\left\{C S_{\tau e}, C S_{\tau}\right\},
$$

where $\mathrm{CS}_{\tau}$ is conditional, is sound and ground-complete for $\mathrm{BCCSP}\left(A_{\tau}\right)$ modulo $\lesssim C S$.

Axiom $C S_{\tau}$ highlights the similarities with the concrete version of complete simulation and with the theory of constrained simulations [10]. However, it is natural to wonder whether it is possible to find a finite, non-conditional and ground-complete axiomatization for $\lesssim C S$ over $\operatorname{BCCSP}\left(A_{\tau}\right)$. Indeed, this is possible; it is enough to substitute the conditional equation $C S_{\tau}$ with the following inequations.

$$
\begin{array}{ll}
(C S) & a x \leq a x+y \\
(\tau N) & \mathbf{0} \leq \tau \mathbf{0}
\end{array}
$$

Theorem 1. The set of unconditional inequations

$$
E_{C S \leq}=B W \cup\left\{C S_{\tau e}, C S, \tau N\right\}
$$

is sound and ground-complete for $\operatorname{BCCSP}\left(A_{\tau}\right)$ modulo $\lesssim C S$. 
It is clear that we could substitute equation $\tau N$ by

$$
(\tau g) \quad x \leq \tau x
$$

in the axiomatization above, since the inequation $\tau g$ is sound for $\operatorname{BCCSP}\left(A_{\tau}\right)$ modulo $\lesssim c s$ and is more general than $\tau N$.

Let us now move on to the ground-complete axiomatization of the largest congruence included in complete simulation equivalence. In order to axiomatize that congruence, it is natural to consider the following equation.

$$
\left(C S E_{\tau}\right) \quad(x \Downarrow \Leftrightarrow y \Downarrow) \Rightarrow a(x+y)=a(x+y)+a x
$$

This equation is essentially the same one that was used in earlier conditional axiomatizations for complete simulation equivalence in the concrete case [10]. However, we remark that the mute predicate deals with silent transitions, although we only use visible actions when describing the equation $C S E_{\tau}$.

Proposition 3. The set of conditional equations

$$
E_{C S}^{c}=B W \cup\left\{C S_{\tau e}, C S E_{\tau}\right\}
$$

is sound and ground-complete for $\operatorname{BCCSP}\left(A_{\tau}\right)$ modulo $\sim_{C S}$.

To turn the previous axiomatization into one without conditional equations we consider the equation

$$
(C S E) \quad a(b x+y+z)=a(b x+y+z)+a(b x+z)
$$

where $a, b \in A$. This is the same equation that is used when axiomatizing complete simulation equivalence in a setting without silent moves.

Theorem 2. The set of unconditional equations

$$
E_{C S}=B W \cup\left\{C S_{\tau e}, C S E\right\}
$$

is sound and ground-complete for $\operatorname{BCCSP}\left(A_{\tau}\right)$ modulo $\approx_{C S}$.

\subsection{Nonexistence of finite complete axiomatizations}

The results in the previous section show that weak complete simulation semantics affords finite (conditional) ground-complete axiomatizations. It is natural to wonder whether the collection of (in)equations over $\operatorname{BCCSP}\left(A_{\tau}\right)$ that are valid in weak complete simulation semantics is finitely axiomatizable.

We shall now prove that if $A$ is non-empty, then the (in)equational theory of $\lesssim C S$ over $\operatorname{BCCSP}\left(A_{\tau}\right)$ does not have a finite basis. (The assumption that $A$ be non-empty is, of course, necessary for such a result. In the trivial case that $A$ is empty, the inequation $x \leq y$ suffices to obtain a complete axiomatization.)

For the sake of clarity, we recall that we consider terms up to the least congruence generated by axioms B1-B4, that is, up to strong bisimilarity. 


\begin{tabular}{|c|c|c|c|c|}
\hline \multirow{2}{*}{$\begin{array}{c}\text { Weak Complete Simulation } \\
\text { Finite Equations }\end{array}$} & \multicolumn{2}{|c|}{ Ground-complete } & \multicolumn{2}{|c|}{ Complete } \\
\hline & Order & Equiv. & Order & Equiv. \\
\hline $1 \leq|A|=\infty$ & $E_{C S} \leq$ & $E_{C S}=$ & \multicolumn{2}{|c|}{ Do not exist } \\
\hline
\end{tabular}

Table 1. Axiomatizations for the largest (pre)congruence included in the weak complete simulation semantics

Our proof of the nonfinite axiomatizability result for the (in)equational theory of $\lesssim C S$ over $\operatorname{BCCSP}\left(A_{\tau}\right)$ will be based on the following infinite family of inequations, which are sound modulo $\lesssim C S$ :

$$
a^{n} x \leq a^{n} \mathbf{0}+a^{n}(x+a) \quad(n \geq 1) .
$$

To see that each of the inequations in the above family is sound, it suffices to observe that if $p \approx_{C S} \mathbf{0}$ then $a^{n} p \lesssim_{C S} a^{n} \mathbf{0}$ for each $n \geq 0$, and $a^{n} p \lesssim_{C S} a^{n}(p+a)$ otherwise, if $n \geq 1$. (Note that the assumption that $n \geq 1$ is necessary for the soundness of the above type of inequation. Indeed, the inequation

$$
x \leq \mathbf{0}+(x+a)
$$

is not sound modulo $\lesssim C S$ because $\mathbf{0} L_{C S} \mathbf{0}+(\mathbf{0}+a)$.)

Theorem 3. If $|A| \geq 1$ then the (in)equational theory of $\lesssim C S$ over $\operatorname{BCCSP}\left(A_{\tau}\right)$ does not have a finite (in)equational basis. In particular, the following statements hold true.

1. No finite set of sound inequations over $\operatorname{BCCSP}\left(A_{\tau}\right)$ modulo $\lesssim C S$ can prove all of the sound inequations in the family

$$
a^{n} x \leq a^{n} \mathbf{0}+a^{n}(x+a) \quad(n \geq 1) .
$$

2. No finite set of sound (in)equations over $\operatorname{BCCSP}\left(A_{\tau}\right)$ modulo $\lesssim C S$ can prove all of the sound equations in the family

$$
a^{n} x+a^{n} \mathbf{0}+a^{n}(x+a)=a^{n} \mathbf{0}+a^{n}(x+a) \quad(n \geq 1) .
$$

Theorem 3 is a corollary of the following result.

Proposition 4. Assume that $|A| \geq 1$. Let $E$ be a collection of inequations whose elements are sound modulo $\lesssim_{C S}$ and have depth smaller than $n$. Suppose furthermore that the inequation $t \leq u$ is derivable from $E$ and that $u \precsim C S a^{n} \mathbf{0}+a^{n}(x+a)$. Then $t \stackrel{a^{n}}{\Longrightarrow} x$ implies $u \stackrel{a^{n}}{\Longrightarrow} x$.

Having shown the above result, statement 1 in Theorem 3 can be proved as follows. Let $E$ be a finite inequational axiom system that is sound modulo $\lesssim$ Cs. Pick $n$ larger than the depth of any axiom in $E$. Then, by Proposition $4, E$ cannot prove the valid inequation

$$
a^{n} x \leq a^{n} \mathbf{0}+a^{n}(x+a),
$$




\begin{tabular}{|l|ll|}
\hline \multicolumn{3}{|c|}{ Unconditional } \\
\hline & $\left(C S_{\tau e}\right)$ & $\tau(a x+y)=a x+y$ \\
$E_{C S \leq}=B W \cup\left\{C S_{\tau e}, C S, \tau N\right\}$ & $(C S)$ & $a x \leq a x+y$ \\
$E_{C S}=B W \cup\left\{C S_{\tau e}, C S E\right\}$ & $(\tau N)$ & $0 \leq \tau \mathbf{0}$ \\
& $(C S E)$ & $b(a x+y+z)=$ \\
& & $b(a x+y+z)+b(a x+z)$ \\
\hline \multicolumn{3}{|c|}{ Conditional } \\
\hline$E_{C S \leq}^{c}=B W \cup\left\{C S_{\tau e}, C S_{\tau}\right\}$ & $\left(C S_{\tau}\right)$ & $(x \Downarrow \Leftrightarrow y \Downarrow) \Rightarrow x \leq x+y$ \\
$E_{C S=}^{c}=B W \cup\left\{C S_{\tau e}, C S E_{\tau}\right\}$ & $\left(C S E_{\tau}\right)$ & $(x \Downarrow \Leftrightarrow y \Downarrow) \Rightarrow$ \\
& & $a(x+y)=a(x+y)+a x$ \\
\hline
\end{tabular}

Table 2. Axioms for the largest (pre)congruence included in the weak completed simulation semantics

and is therefore incomplete. Indeed,

$$
a^{n} x \stackrel{a^{n}}{\Longrightarrow} x
$$

On the other hand, the only terms $t$ such that

$$
a^{n} \mathbf{0}+a^{n}(x+a) \stackrel{a^{n}}{\Longrightarrow} t
$$

holds are $\mathbf{0}$ and $x+a$. So $a^{n} \mathbf{0}+a^{n}(x+a) \stackrel{a^{n}}{\Longrightarrow} x$ does not hold.

Corollary 1. If $|A| \geq 1$ then the collection of (in)equations in at most one variable that hold over $\operatorname{BCCSP}\left(A_{\tau}\right)$ modulo $\lesssim C S$ does not have a finite (in)equational basis. Moreover, for each $n$, the collection of all sound (in)equations of depth at most $n$ cannot prove all the valid (in)equations in at most one variable that hold in weak complete simulation semantics over $\operatorname{BCCSP}\left(A_{\tau}\right)$.

Tables 1-2 summarizes the positive and negative results on the existence of finite axiomatizations for weak complete simulation semantics. On Table 1, 'Do not exist' indicates that there is no finite (in)equational axiomatization for the corresponding semantic relation.

\section{Conclusion}

In this paper, we have offered a detailed study of the equational theory of the largest precongruence and congruence over the language BCCSP induced by the weak version of the classic complete simulation preorder and equivalence, respectively. 
On the one hand, for these (pre)congruence we have presented results that show the existence of finite ground-complete equational axiomatizations. In order to obtain a better understanding of the equational theory of weak complete simulation semantics, we have presented both conditional and unconditional versions of such axiomatizations.

On the other hand, we have proved that the (in)equational theory of the weak complete simulation precongruence over BCCSP does not have a finite (in)equational basis. This result is true in the presence of at least one observable action. Moreover, we have shown that the collection of (in)equations in at most one variable that hold in weak complete simulation semantics over BCCSP does not have an (in)equational basis of bounded depth, and therefore not a finite one.

It would be interesting to obtain infinite, but finitely described, complete axiomatizations of weak complete simulation semantics. This is a topic that we leave for future research.

While in [2] we showed that for both weak simulation and weak ready simulation semantics the finite axiomatizability of the semantics was crucially dependent on whether the set of observable actions was finite or infinite, for the weak complete simulation semantics this is not the case. As reflected in Table 1, for any non-empty set of actions, it is impossible to find a finite complete axiomatization.

We find it pleasing that all the known results on the existence of finite bases for the weak, complete and plain simulation semantics (see [8]) in the 'concrete' case, that is without silent moves, are 'lifted' to the weak version of the simulation semantics we have presented.

We plan to investigate now the weak versions of semantics in van Glabbeek's spectrum that are based on notions of decorated traces. We have already started working on this topic and we plan to report on our results in a forthcoming article.

Following the developments in $[1,5,21]$, it would also be interesting to study rule formats for operational semantics that provide congruence formats for the semantics considered in this paper, and to give procedures for generating ground-complete axiomatizations for them for process languages in the given formats.

\section{References}

1. L. Aceto, B. Bloom, and F. W. Vaandrager. Turning SOS rules into equations. Information and Compututation, 111(1):1-52, 1994.

2. L. Aceto, D. de Frutos-Escrig, C. Gregorio-Rodríguez, and A. Ingólfsdóttir. Axiomatizing weak ready simulation semantics over BCCSP. In A. Cerone and P. Pihlajasaari, editors, Proceedings of the 8th International Colloquium on Theoretical Aspects of Computing, ICTAC 2011, Lecture Notes in Computer Science. Springer-Verlag, 2011. To appear.

3. L. Aceto, W. Fokkink, A. Ingólfsdóttir, and B. Luttik. Finite equational bases in process algebra: Results and open questions. In Processes, Terms and Cycles, volume 3838 of Lecture Notes in Computer Science, pages 338-367. Springer, 2005. 
4. J. Baeten, T. Basten, and M. Reniers. Process Algebra: Equational Theories of Communicating Processes, volume 50 of Cambridge Tracts in Theoretical Computer Science. Cambridge University Press, November 2009.

5. J. C. M. Baeten and E. P. de Vink. Axiomatizing GSOS with termination. Journal of Logic and Algebraic Programming, 60-61:323-351, 2004.

6. J. A. Bergstra and J. W. Klop. Process algebra for synchronous communication. Information and Control, 60(1-3):109-137, 1984.

7. B. Bloom, S. Istrail, and A. R. Meyer. Bisimulation can't be traced. Journal of the ACM, 42(1):232-268, 1995.

8. T. Chen, W. Fokkink, B. Luttik, and S. Nain. On finite alphabets and infinite bases. Information and Computation, 206(5):492-519, 2008.

9. D. de Frutos-Escrig, C. Gregorio-Rodríguez, and M. Palomino. On the unification of process semantics: Equational semantics. Electronic Notes in Theoretical Computer Science, 249:243-267, 2009.

10. D. de Frutos-Escrig and C. Gregorio-Rodríguez. Universal coinductive characterizations of process semantics. In 5th IFIP International Conference on Theoretical Computer Science, volume 273 of IFIP, pages 397-412. Springer, 2008.

11. R. v. Glabbeek. The linear time - branching time spectrum I; the semantics of concrete, sequential processes. In Handbook of Process Algebra, chapter 1, pages 3-99. Elsevier, 2001.

12. M. Hennessy and R. Milner. Algebraic laws for nondeterminism and concurrency. Journal of the ACM, 32:137-161, 1985.

13. C. Hoare. Communicating Sequential Processes. Prentice Hall, 1985.

14. K. G. Larsen and A. Skou. Bisimulation through probabilistic testing. Information and Computation, 94(1):1-28, 1991

15. R. Milner. An algebraic definition of simulation between programs. In Proceedings 2nd Joint Conference on Artificial Intelligence, pages 481-489. BCS, 1971. Also available as Report No. CS-205, Computer Science Department, Stanford University.

16. R. Milner. Communication and Concurrency. Prentice Hall, 1989.

17. R. Milner. A complete axiomatisation for observational congruence of finite-state behaviors. Information and Computation, 81(2):227-247, 1989.

18. F. Moller. Axioms for Concurrency. PhD thesis, Report CST-59-89, Department of Computer Science, University of Edinburgh, 1989.

19. D. M. Park. Concurrency and automata on infinite sequences. In Theoretical Computer Science, 5th GI-Conference, volume 104 of Lecture Notes in Computer Science, pages 167183. Springer, 1981.

20. G. D. Plotkin. A structural approach to operational semantics. Journal of Logic and Algebraic Programming, 60-61:17-139, 2004.

21. I. Ulidowski. Axiomatisations of weak equivalences for De Simone languages. In CONCUR '95: Concurrency Theory, 6th International Conference, volume 962 of Lecture Notes in Computer Science, pages 219-233. Springer, 1995. 